\title{
Theoretical and Empirical Estimates of V-index Variability
}

\author{
Massimo W Rivolta ${ }^{1}$, Luca T Mainardi ${ }^{1}$, Roberto Sassi ${ }^{1}$ \\ ${ }^{1}$ Dipartimento di Informatica, Università degli Studi di Milano, Italy \\ ${ }^{2}$ Dipartimento di Bioingegneria, Politecnico di Milano, Italy
}

\begin{abstract}
Spatial heterogeneity of the ventricular repolarization (SHVR) might lead to life-threatening events when pathologically increases. The V-index is a metric meant to assess SHVR from the surface ECG and is computed on a set of ECG beats. The number of beats required to ensure low variability estimates is still matter of investigation. In this study, we investigated the role of the variability of the $V$-index estimator as function of the number of beats, using computerized simulations. We generated 100 sets of 1000 beats with seven different values of SHVR (from 10 to $70 \mathrm{~ms}$ ) using the ECGSIM model. Using such dataset, we tested two approaches to estimate the variability of the $V$-index estimator. First, we analytically derived the probability density function of the estimator under independence and normality assumptions. Second, we used the bootstrap technique and derived that 1000 bootstrap iterations ensured stable estimates. We obtained that the standard deviation (STD) of V-index increased with SHVR values and decreased with the number of beats considered, with a rate similar to that of the sample mean. The independence assumption overestimated the STD by a factor of about 2. To conclude, the variability of the V-index estimator is below $5 \mathrm{~ms}$ when at least 100 beats are considered. The number of beats is in line with other techniques meant to estimate SHVR.
\end{abstract}

\section{Introduction}

It is well known that the heterogeneity of the ventricular repolarization is responsible for the genesis of the T-wave on the ECG and might lead to life-threatening events when pathologically increased. Intense effort has been put in play to understand the underlying mechanisms of such increased heterogeneity and to provide indexes, computable from the ECG, capable to prevent cardiac events or to perform risk stratification.

Among the indexes developed in the last decades [1], the $\mathrm{V}$-index is meant to assess the spatial heterogeneity of the ventricular repolarization (SHVR) from the surface ECG [2]. The index was proven to detect: i) pathological condi- tions such as those in patients suffering from Chagas disease [3] or in patients with symptoms suggestive of nonST-elevation myocardial infarction [4]; and ii) effects of administration of pro-arrhythmic drugs $[5,6]$.

The index requires a set of ECG beats to be computed. However, the number of beats required to ensure low variability estimates is still matter of investigation. Such variability can be decomposed into three factors: i) the intrasubject variability; ii) the variability due to the estimation algorithms; and iii) the variability of the estimator itself. In this study, we investigated on the role of the last factor as function of the number of beats. Specifically, we provided two methodologies to estimate the variability of the V-index estimator and tested them on synthetic simulations.

\section{Methods}

\subsection{Background on the V-index}

The V-index is derived by plugging together a forward model of the surface ECG [7] and a statistical model of the repolarization times [2]. Briefly, the surface ECG is modelled by a linear combination of the transmembrane potential of the cardiac myocites across the heart surface. The model is as follows

$$
\boldsymbol{\Psi}(\mathbf{t})=\mathbf{A}\left[\begin{array}{c}
D\left(t-\rho_{1}\right) \\
\cdots \\
D\left(t-\rho_{M}\right)
\end{array}\right]
$$

where $t$ is time, $D(t)$ is a single transmembrane potential shared (only during repolarization) by all the $M$ myocytes, $\rho_{m}$ is the repolarization time of the $m$-th myocite (for example the time of the steepest downslope of $D(t)), A$ is the subject-dependent $L \times M$ transfer matrix where $L$ is the number of leads, and $\boldsymbol{\Psi}(\mathbf{t})$ is a $L \times 1$ vector of the surface ECG.

The statistical model describes the dispersion of the repolarization times as follows

$$
\Delta \rho_{m}=\rho_{m}-\bar{\rho}=\theta_{m}+\phi_{m}(k)
$$

where $\bar{\rho}$ is the average repolarization time, $\theta_{m}$ is the repolarization time for a specific myocite, $k$ is the beat number 
and $\phi_{m}(k)$ is the random beat-to-beat variation. $\phi_{m}(k)$ is typically heart-rate dependent.

The model in eq. (1) can be decomposed using a Taylor expansion obtaining

$$
\begin{aligned}
\mathbf{\Psi}(t) & =\sum_{i=1}^{P} \mathbf{w}_{\mathbf{i}} \frac{d^{i-1}}{d t^{i-1}} T_{d}(t)=\ldots \\
\ldots & =\mathbf{w}_{\mathbf{1}} T_{d}(t)+\mathbf{w}_{\mathbf{2}} \dot{T}_{d}(t)+\mathbf{E}(t)
\end{aligned}
$$

where $P$ is the number of terms in the expansion, $\mathbf{w}_{\mathbf{i}}$ are vectors of constant values called lead factors, $T_{d}(t)=$ $-\dot{D}(t)$ is the dominant T-wave, $\mathbf{w}_{\mathbf{1}}=-A \boldsymbol{\Delta} \rho(\boldsymbol{\Delta} \rho$ is the $M \times 1$ vector containing all $\Delta \rho_{m}$ values), $\mathbf{w}_{\mathbf{2}}=\frac{1}{2} A \boldsymbol{\Delta} \rho^{2}$ and $\mathbf{E}(t)$ comprises all the higher order terms.

It is possible to show that the standard deviation (STD) of $\theta$ across myocites in a single beat can be approximated as follows

$$
s_{\theta}=\left(\frac{\sum_{m=1}^{M} \theta_{m}^{2}}{M}\right)^{\frac{1}{2}} \approx \frac{\operatorname{STD}\left[w_{2}(l)\right]}{\operatorname{STD}\left[w_{1}(l)\right]}
$$

where $l$ is the lead number and the STD of the lead factors is computed across beats. The quantity returns $L$ estimates of $s_{\theta}$ and the $\mathrm{V}$-index is defined as their average.

In practice, the $\mathrm{V}$-index is estimated using the following formulas

$$
\begin{aligned}
\bar{w}_{1}(l) & =\frac{1}{B} \sum_{k=1}^{B} \hat{w}_{1}(l, k), \bar{w}_{2}(l)=\frac{1}{B} \sum_{k=1}^{B} \hat{w}_{1}(l, k) \\
\hat{V}(l) & =\sqrt{\frac{\sum_{k=1}^{B}\left(\hat{w}_{2}(l, k)-\bar{w}_{2}(l)\right)^{2}}{\sum_{b=1}^{B}\left(\hat{w}_{1}(l, b)-\bar{w}_{1}(l)\right)^{2}}}
\end{aligned}
$$

where $\hat{w}_{1}(l, k)$ and $\hat{w}_{2}(l, k)$ are the first and second estimated lead factors of lead $l$ and beat $k, B$ is the number of beats and $\hat{V}(l)$ is the estimated $\mathrm{V}$-index for the lead $l$. The final $\mathrm{V}$-index estimate is computed by averaging the $L$ values of $\hat{V}(l)$.

\subsection{Variability of the $\mathrm{V}$-index}

We propose two methodologies to estimate the variability of the V-index estimator.

The first methodology assumes that $\hat{w}_{1}$ and $\hat{w}_{2}$ are normally distributed and independent between each other. In our experiments, the normality assumption appears to hold when the V-index is computed under stable conditions of the patient, for example, when the ECG is collected during rest. Here, we modeled these two quantities using a rescaled version of the standard Normal distribution, as follows

$$
\begin{aligned}
& \hat{w}_{1}=\sigma_{1} z_{1}+\mu_{1} \\
& \hat{w}_{2}=\sigma_{2} z_{2}+\mu_{2}
\end{aligned}
$$

where $z_{1}$ and $z_{2}$ are two standard Normal variables.

When the value $\mu_{1}$ and $\mu_{2}$ are known, i.e., $\bar{w}_{1}=\mu_{1}$ and $\bar{w}_{2}=\mu_{2}$, the square of the ratio in eq. (5) becomes a rescaled version of the ratio of two $\chi^{2}$ distributions with $B$ degrees of freedom each. Without lack of generality, by considering one of the $l$ variable from eq. (5), we obtain that

$$
\hat{V}^{2}=\frac{\sigma_{2}^{2}}{\sigma_{1}^{2}} \frac{\sum_{k=1}^{B} z_{2}^{2}(k)}{\sum_{b=1}^{B} z_{1}^{2}(b)}=\frac{\sigma_{2}^{2}}{\sigma_{1}^{2}} f
$$

where $f$ follows the $F(B, B)$ distribution.

The distribution of the square root of the previous equation can be derived by change of variable, leading to

$$
g_{\hat{V}}(v)=2 \frac{\sigma_{1}^{2}}{\sigma_{2}^{2}} g_{F}\left(\frac{\sigma_{1}^{2}}{\sigma_{2}^{2}} v^{2}\right) v
$$

where $g_{V}$ and $g_{F}$ are the distribution of the variables $v$ and $f$, respectively. From this equation, the STD can be derived by numerical integration using the standard definition of statistical moments.

The second methodology makes use of the bootstrap technique. Here, in a numerical simulation described in the next section, we randomly selected ECG beats from our dataset. We used a resampling strategy with replacement to estimate the STD of $\hat{V}$ through the $\hat{w}_{1}$ and $\hat{w}_{2}$ values of the selected beats. In this case, the number of bootstraps is a degree of freedom that needs to be set in advance.

\subsection{Synthetic dataset}

The synthetic dataset comprised 100 sets of 1000 ECG beats (12 leads; sampling rate of $1000 \mathrm{~Hz}$ ) generated with a direct electrophysiological model (ECGSIM) [8] for seven values of SHVR in the range 10-70 ms (step $10 \mathrm{~ms}$ ). The forward model had $M=257$ nodes and the repolarization time $\theta_{m}$ was provided for each node $m . \phi_{m}(k)$ values were extracted from a zero-mean Normal distribution with $\sigma_{\phi}=1 \mathrm{~ms}$. In this case, the values of $\hat{w}_{1}$ and $\hat{w}_{2}$ for each ECG beat were constructed using the equations described into sec. 2.1.

This dataset allowed to known the distribution of the "true" V-index value (even though approximated), as function of the number of beats.

\subsection{Analysis}

Three analysis were performed on the synthetic dataset.

First, the STD of the "true" V-index was estimated as a function of the number of beats. We computed the STD of the $100 \mathrm{~V}$-index values when considering the first $B$ beats generated, with $B$ ranging from 50 to 1000 , and for each of the seven values of SHVR. We also derived the slope of 
(a)

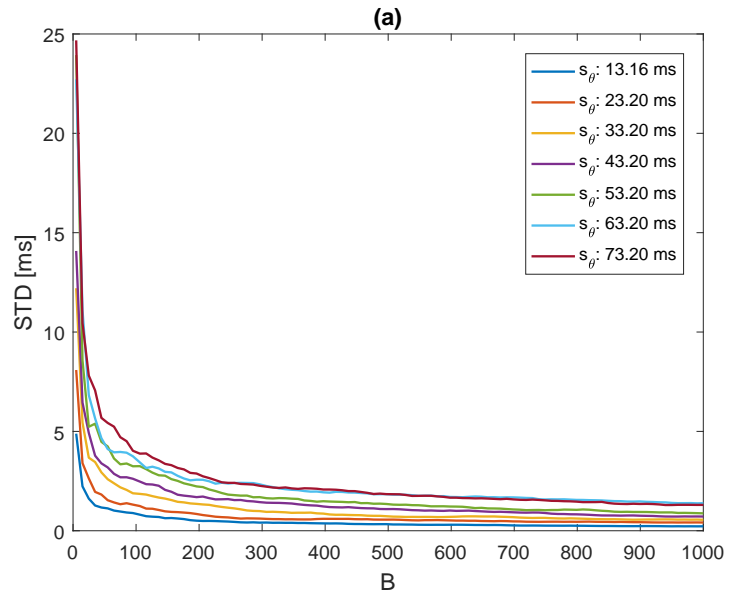

(b)

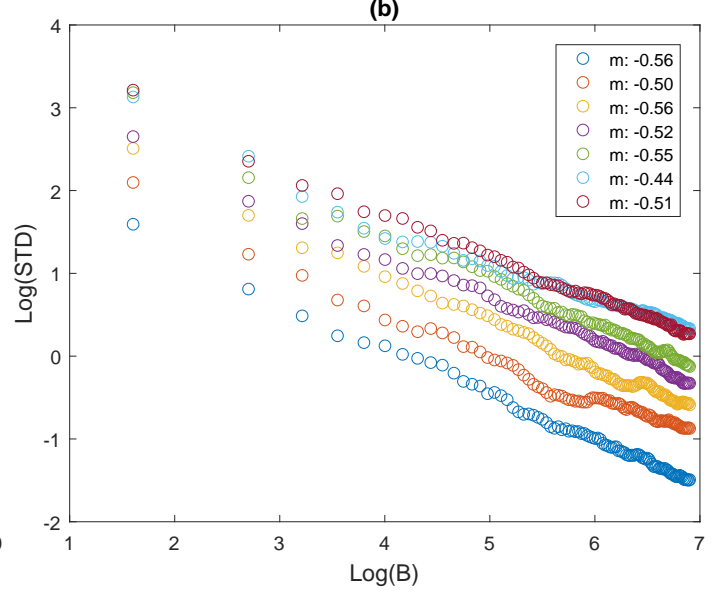

Figure 1. Estimated STD of V-index for increasing number of available beats $B$ and different $s_{\theta}$ values (a). Log-log plot of the STD and $B(\mathrm{~b})$. The estimated slope $m$ is reported inside the legend.

the scatter plot in the log-log scale to compare the rate of convergence with that one of the sample mean, i.e., -0.5 .

Second, we determined the ratio between the STD of the "true" V-index and the STD computed using eq. (8) with numerical integration, as function of the number of beats. The value of $\sigma_{1}$ was set to 1 and $\sigma_{2}$ was set to "true" $\mathrm{V}$-index value for each of the 100 sets. The analysis was repeated for each of the seven SHVR value.

Third, the bootstrap technique was employed to estimate the STD. For each of the 100 sets, we re-sampled the first $B$ beats $T$ times, computed $T \mathrm{~V}$-index values, and estimated the STD. In this way, we obtained the average and standard deviation across 100 possible sets of the STD, for each of the seven SHVR values. Here, we manually identified what $T$ value would provide stable estimates.

\section{Results}

The results of the first analysis are reported in fig. 1. In particular, fig. 1a reports the STD for each of the seven SHVR values when increasing the number of beats in-

Table 1. Ratio between the STD of V-index from ECGSIM and the one computed with the model in eq. (8).

\begin{tabular}{cccc}
\hline \hline SHVR [ms] & $B=100$ & $B=500$ & $B=1000$ \\
\hline 13.16 & 0.59 & 0.54 & 0.52 \\
23.20 & 0.50 & 0.52 & 0.55 \\
33.20 & 0.54 & 0.47 & 0.51 \\
43.20 & 0.51 & 0.55 & 0.51 \\
53.20 & 0.55 & 0.55 & 0.51 \\
63.20 & 0.46 & 0.63 & 0.68 \\
73.20 & 0.51 & 0.54 & 0.55 \\
\hline \hline
\end{tabular}

volved in the computation. We observed that the STD was high for small number of beats and rapidly decreased when a larger number of beats $B$ was considered. Moreover, the higher the SHVR, the higher was the STD. The observation on the rate of convergence was more visible on the log-log plot reported in fig. 1b. Indeed, the slope of the scatter plot was around -0.5 for all the seven SHVR, suggesting a rate of convergence similar to the one of the sample mean.

In the second analysis, we computed the ratio between the STD of the V-index derived from the ECGSIM data and the one estimated using eq. (8) through numerical integration. The ratio was approximately constant at 0.5 for all the seven SHVR values and number of beats $B$ considered. This result suggested that the independence assumption of $\hat{w}_{1}$ and $\hat{w}_{2}$ provided an overestimate of the STD of a factor of about 2. Table 1 reports the ratios computed for the seven SHVR values and $B=50, B=500$ and $B=1000$.

The last analysis aimed to determine a suitable bootstrap number $T$ that would ensure stable estimates of the STD. We obtained a stable estimate using a minimum value $T$ of 1000 , regardless the number of beats involved and SHVR. Figure 2 reports the variability of the STD computed via bootstraps for different values of $B$ and $T$, and two values of SHVR.

\section{Discussion and Conclusion}

In this study, we investigated the variability of the Vindex estimator. We found that the STD was below $5 \mathrm{~ms}$ when at least 100 beats were used. This result was valid for the physiological range of SHVR considered (fig. 1a) generated using the ECGSIM model.

We tackled the problem of estimating the variability using two approaches. The first one assumed independence and normality of the lead factors. This approach led to 

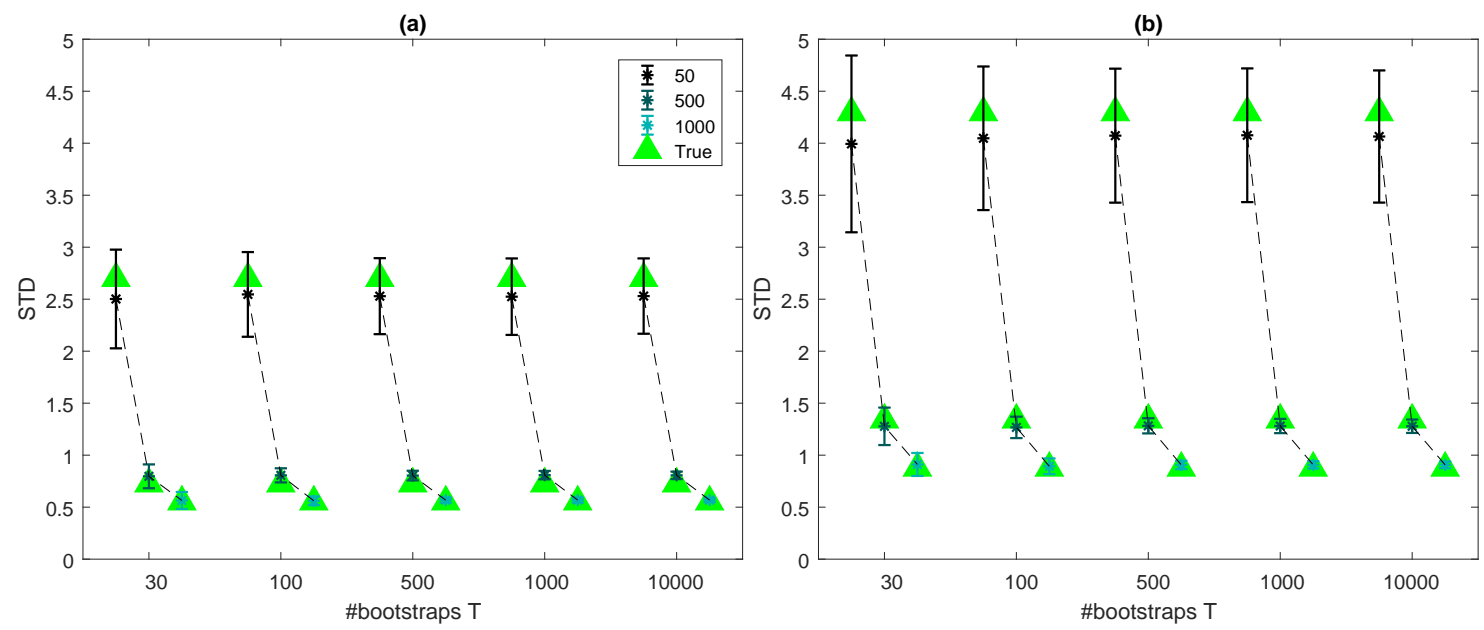

Figure 2. STD estimated through bootstrap for several values of the number of beats $B$ and number of bootstraps $T$. Two values of SHVR are reported, i.e., $32.20 \mathrm{~ms}$ (a) and $52.20 \mathrm{~ms}$ (b). The vertical bars indicate the STD of the bootstrap estimate across the 100 sets. The green triangle refers to the value directly computed from the ECGSIM data while the dashed lines indicate the increase of the number of beat considered for a given $T$.

produce an overestimate of the variability by a factor of about 2 (tab. 1), regardless the SHVR value. This technique set an upper boundary on the variability of the Vindex estimator and can be computed just considering the value of V-index and number of beats $B$. Knowing such upper boundary might be useful in different circumstances like providing an estimate of the estimator variability on short recordings or to deploy more reliable V-index estimation algorithms. Another important finding was that the variability increased with the SHVR value and the ratio between the variabilities computed with the model in eq. (8) and from the ECGSIM data was constant (tab. 1).

Using the second approach (bootstrapping), we found that at least 1000 resampling were enough to produce a stable estimate of the V-index. This result was valid for all the considered SHVR and number of beats $B$ (fig. 2). This technique can be also used on real data but it needs further investigation since noise and estimation algorithms might play a role.

To conclude, independently from SHVR in the physiological range, the variability of the V-index estimator is below $5 \mathrm{~ms}$ when at least 100 beats are considered. Such number of beats is in line with the amount required by other techniques meant to estimate SHVR [1].

\section{References}

[1] Laguna P, Martínez JP, Pueyo E. Techniques for ventricular repolarization instability assessment from the ecg. Proc IEEE 2016;104(2):392-415.

[2] Sassi R, Mainardi LT. An estimate of the dispersion of repolarization times based on a biophysical model of the ECG. IEEE Trans Biomed Eng 2011;58(12):3396-3405.

[3] Sassi R, Rivolta MW, Mainardi LT, Reis RC, Rocha MOC,
Ribeiro ALP, Lombardi F. Spatial Repolarization Heterogeneity and Survival in Chagas Disease. Methods Inf Med 2014;53(4):1-5.

[4] Abächerli R, Twerenbold R, Boeddinghaus J, Nestelberger T, Mächler P, Sassi R, Rivolta MW, Roonizi EK, Mainardi LT, Kozhuharov N, Rubini Giménez M, Wildi K, Grimm K, Sabti Z, Hillinger P, Puelacher C, Strebel I, Cupa J, Badertscher P, Roux I, Schmid R, Leber R, Osswald S, Mueller C, Reichlin T. Diagnostic and prognostic values of the $\mathrm{V}$-index, a novel ECG marker quantifying spatial heterogeneity of ventricular repolarization, in patients with symptoms suggestive of non-ST-elevation myocardial infarction. Int J Cardiol 2017;236:23-29.

[5] Rivolta MW, Mainardi LT, Sassi R. Quantification of ventricular repolarization heterogeneity during moxioxacin or sotalol administration using V-index. Physiol Meas 2015; 36(4):803-811.

[6] Corino VDA, Rivolta MW, Mainardi LT, Sassi R. Assessment of spatial heterogeneity of ventricular repolarization after quinidine in healthy subjects. Comput Cardiol 2017; 44:1-4.

[7] van Oosterom A. Genesis of the $T$ wave as based on an equivalent surface source model. J Electrocardiol 2001;34 Suppl:217-227.

[8] van Oosterom A. The dominant T wave. J Electrocardiol 2004;37:193-197.

Address for correspondence:

Massimo W. Rivolta

Dipartimento di Informatica / Università degli Studi di Milano

Via Bramante 65 / Crema / Italy

tel: +390250330056

massimo.rivolta@unimi.it 